Vol. 137 (1995), 55-75

\title{
THE CONTINUITY PRINCIPLE IN EXPONENTIAL TYPE ORLICZ SPACES
}

\author{
S. E. GRAVERSEN, G. PEŠKIR AND M. WEBER
}

\section{Introduction}

In this section we shall present some facts in the background of the problem under our next consideration. Let $(X, \mathscr{A}, \mu)$ be a finite measure space, and let $B$ be a Banach space with a norm $\|\cdot\|$. Let $M(\mu)$ denote the linear space of all $\mu$-measurable functions from $X$ into $\mathbf{R}$, and let $T$ be a linear operator from $B$ into $M(\mu)$. Then $T$ is said to be continuous in $\mu$-measure, if $\left\|f_{n}-f\right\| \rightarrow 0$ implies $\mu\left\{\left|T f_{n}-T f\right|>\varepsilon\right\} \rightarrow 0$ as $n \rightarrow \infty$, for all $\varepsilon>0$ with $f_{n}$ and $f$ in $B$ for $n \geq 1$. If $\left\{T_{n} \mid n \geq 1\right\}$ is a sequence of linear operators from $B$ into $M(\mu)$, then we denote $\left(T^{*} f\right)(x)=\sup _{n \geq 1}\left|\left(T_{n} f\right)(x)\right|$ for $f \in B$ and $x \in X$. It is well-known that we have:

(1.1) (Banach's principle)

Let $\left\{T_{n} \mid n \geq 1\right\}$ be a sequence of linear operators from $B$ into $M(\mu)$ such that every $T_{n}$ is continuous in $\mu$-measure for $n \geq 1$. Then $T^{*} f<\infty \mu$-a.e. for all $f \in B$, if and only if there exists a decreasing function $C: \mathbf{R}_{+} \rightarrow \mathbf{R}_{+}$ satisfying $\lim _{\lambda \rightarrow \infty} C(\lambda)=0$ such that we have:

$$
\mu\left\{T^{*} f>\lambda\|f\|\right\} \leq C(\lambda)
$$

for all $\lambda>0$ and all $f \in B$.

A main implicit usefulness of Banach's principle follows from the well-known fact that having a sequence of linear operators $\left\{T_{n} \mid n \geq 1\right\}$ from $B$ into $M(\mu)$ satisfying the inequality in (1.1) the set of all $f \in B$ for which the sequence of functions $\left\{T_{n} f \mid n \geq 1\right\}$ converges $\mu$-a.e. is closed in $B$, see [5] (p.3). In this way the establishment of a.e.-convergence over the whole space $B$ very often becomes equivalent to the establishment of the inequality in (1.1). Another natural question is to determine the explicit form of the function $C$ appearing in (1.1). Under our general hypotheses on the operators and spaces in (1.1) it is difficult to believe in a possibility of deducing any further information on $C$ besides the given one.

Received August 31, 1992. 
However more informative inequalities in some particular cases are obtained a long time ago by Kolmogorov in [8] and Calderon in [19]. Finally they were resulted by Stein's continuity principle giving a specific form to the function $C$ under additional hypotheses which may be described as follows:

(1.2) The Banach space $B$ equals $L^{p}(\mu)$ with $1 \leq p \leq 2$.

(1.3) There exists a family $\mathscr{E}$ of measure-preserving transformations in $(X, \mathscr{A}, \mu)$ which is "mixing" in the following sense: For any $A, B \in \mathscr{A}$ with $\mu(A)$. $\mu(B)>0$ and any $\alpha>1$, there exists $E \in \mathscr{E}$ such that:

$$
\mu(X) \cdot \mu\left(A \cap E^{-1}(B)\right) \leq \alpha \cdot \mu(A) \mu(B) .
$$

(1.4) The sequence $\left\{T_{n} \mid n \geq 1\right\}$ and the family $\mathscr{E}$ "commute" in the following sense:

$$
E\left(T^{*} f\right) \leq T^{*}(E f)
$$

for all $f \in B$ and all $E \in \mathscr{E}$.

(1.5) (Stein's continuity principle)

Under (1.2)-(1.4) we have $T^{*} f<\infty \mu$-a.e. for all $f \in L^{p}(\mu)$, if and only if:

$$
\mu\left\{T^{*} f>\lambda\right\} \leq \frac{C_{p}}{\lambda^{p}} \int|f|^{p} d \mu
$$

for all $\lambda>0$ and all $f \in L^{p}(\mu)$ with some $C_{p}>0$.

Actually Sawyer was who recognized the above hypotheses and the non-essence of a group theoretic setting as it was considered by Stein himself. He has also observed that the above principle remains valid for $p>2$ if the operators $T_{n}$ are assumed to be positive, that is $T_{n} f \geq 0 \mu$-a.e. whenever $f \geq 0 \mu$-a.e. with $f \in$ $L^{p}(\mu)$ and $n \geq 1$. So we have:

(1.6) (Sawyer's continuity principle)

If every $T_{n}$ in (1.5) is assumed to be positive for $n \geq 1$, then the equivalence in (1.5) remains valid for all $1 \leq p \leq \infty$.

Methods used in the proofs of principles (1.5) and (1.6) strongly rely upon Banach's principle (1.1) with an instructive and nice application of the Rademacher randomization in Stein's case. For more details in this direction we shall refer the reader to [5] (p. 1-15).

The main purpose of this paper is to investigate and establish a continuity principle of Sawyer's type for positive linear operators in the case where $B$ equals 
to the Orlicz space $L^{\psi_{p}}(\mu)$ with $\phi_{p}(t)=\exp \left(t^{p}\right)-1$ for $t \geq 0$ and $1 \leq p<\infty$. A very instructive observation in this direction may be obtained by a reformulation of the inequality in (1.5) and (1.6) in the following way:

$$
\sup _{\lambda>0}\left(\lambda^{p} \cdot \mu\left\{T^{*} f>\lambda\right\}\right)^{1 / p} \leq C_{p} \cdot\left(\int|f|^{p} d \mu\right)^{1 / p} .
$$

Then one might recognize the left side above as a Laurent norm of $T^{*} f$ induced by the function $\varphi_{p}(t)=t^{p}$ for $t \geq 0$, while the $L^{p}$-norm of $f$ on the right side may be seen as the Orlicz norm of $f$ induced by the same function $\varphi_{p}$, see [7]. The given Laurent norm is often called the weak $L^{p}$-norm and is denoted by $\|\cdot\|_{p, \infty}$. Therefore the inequality in (1.7) may be equivalently written as follows:

$$
\left\|T^{*} f\right\|_{p, \infty} \leq C_{p} \cdot\|f\|_{p} .
$$

Since the given Laurent and Orlicz norms are induced by the same functions $\varphi_{p}$ we may follow the general terminology involving some other suitable functions $\varphi$, and say that they are associated each to other. Moreover it is well-known and easily verified that a given Laurent norm is always less or equal to the associated Orlicz norm, see [7] (p. 11). In particular we have:

$$
\left\|T^{*} f\right\|_{p, \infty} \leq\left\|T^{*} f\right\|_{p} .
$$

Furthermore it is well-known that the left side in (1.8) cannot be replaced by the right side in (1.9) in general. However in the case where we consider exponential $\varphi_{p}$ instead of polynomial $\varphi_{p}$ the induced Laurent norms become equivalent to the associated Orlicz norms, see section 2 . Therefore the inequality which corresponds to that given in (1.5) and (1.6) clearly reduces to the following one:

$$
\left\|T^{*} f\right\|_{\psi_{p}} \leq C_{p} \cdot\|f\|_{\psi_{p}}
$$

being valid for all $f \in L^{\psi_{p}}(\mu)$ with $1 \leq p<\infty$. Here $\|\cdot\|_{\psi_{p}}$ denotes the Orlicz norm induced by $\phi_{p}$ for $1 \leq p<\infty$. Thus we shall be in the next mainly concerned on the establishment of inequality (1.10) having in mind the equivalence between the Orlicz norm of $T^{*} f$ on the left side in (1.10) and the associated Laurent norm of $T^{*} f$ which is given by:

$$
\left\|T^{*} f\right\|_{\phi_{p}, \infty}=\sup _{\lambda>0}\left(\lambda^{p} / \log \left(1+\frac{1}{\mu\left\{T^{*} f>\lambda\right\}}\right)\right)^{1 / p}
$$

Since the right side above contains the tail $\mu$-measure of $T^{*} f$, it is quite clear that inequality (1.10) will also contain all needed information concerning the specific form of the function $C$ arising in Banach's principle in this case. Another interest- 
ing reason for this investigation is coming from the fact that inequality (1.10) may be viewed as a dominated ergodic theorem in the Orlicz space $L^{\psi_{p}}(\mu)$ for $1 \leq p$ $<\infty$, at least when the operators under consideration have the average form. We shall however see that in this case inequality (1.10) may be established in a rather elementary way by using the well-known dominated ergodic theorems in $L^{p}$-spaces, but this technique does not apply for more general operators. To conclude this introduction let us say that the method used in the proof of our main result relies upon Banach's principle and a technique established in Stein-Sawyer's continuity principles in $L^{p}$-spaces.

\section{Preliminary facts}

In this section we shall recall some definitions and more or less known facts on the objects under next consideration. We begin by considering Orlicz and Laurent spaces. Let $(X, \mathscr{A}, \mu)$ be a finite measure space, and let $\varphi$ be an increasing left continuous function from $\left[0, \infty\left[\right.\right.$ into $\left[0, \infty\left[\operatorname{such}\right.\right.$ that $\varphi(0)=\lim _{t \downarrow 0} \varphi(t)=$ 0 . Then we may consider the following Orlicz norm induced by $\varphi$, see [7]:

$$
\|f\|_{\tau_{\varphi}}=\inf \left\{a>0 \mid \int^{*} \varphi\left(a^{-1}|f|\right) d \mu \leq 1\right\}
$$

whenever $f$ belongs to $\mathbf{R}^{X}$ with inf $\emptyset=\infty$. Recall that $\int^{*} g d \mu$ denotes the upper $\mu$-integral of a functlon $g$ from $X$ into $\mathbf{R}$. The corresponding Orlicz space is defined by:

$$
L^{\tau_{\varphi}}\left(\mu^{*}\right)=\left\{f \in \mathbf{R}^{X} \mid \lim _{\varepsilon \downarrow 0}\|\varepsilon f\|_{\tau_{\varphi}}=0\right\}
$$

It is well-known that we have:

(2.1) If $\varphi$ is convex, then $\left(L^{\tau_{\varphi}}\left(\mu^{*}\right),\|\cdot\|_{\tau_{\varphi}}\right)$ is a Banach space.

Similarly we may consider the following Laurent norm induced by $\varphi$, see [7]:

$$
\|f\|_{\sigma_{\varphi}}=\inf \left\{a>0 \mid \sup _{t>0}\left(\varphi(t) \cdot \mu^{*}\{|f|>a \cdot t\}\right) \leq 1\right\}
$$

whenever $f$ belongs to $\mathbf{R}^{X}$. Recall that $\mu^{*}$ denotes the outer $\mu$-measure. The corresponding Laurent space is defined by:

$$
\Lambda^{\sigma_{\varphi}}\left(\mu^{*}\right)=\left\{f \in \mathbf{R}^{X} \mid \lim _{\varepsilon \downarrow 0}\|\varepsilon f\|_{\tau_{\varphi}}=0\right\} .
$$

It is well-known that we have: 
(2.2) If $\varphi$ is convex at 0 , i.e. $\varphi(\lambda t) \leq \lambda \varphi(t)$ for all $\lambda \in[0,1]$ and all $t \geq 0$, then $\left(\Lambda^{\sigma_{\varphi}}\left(\mu^{*}\right),\|\cdot\|_{\sigma_{\varphi}}^{1 / 2}\right)$ is a Fréchet space.

In addition we may also consider the following norm and space:

$$
\begin{aligned}
& \|f\|_{0}=\inf \left\{a>0 \mid \mu^{*}\{|f|>a\} \leq a\right\} \\
& L^{0}\left(\mu^{*}\right)=\left\{f \in \mathbf{R}^{X} \mid \lim _{\varepsilon \downarrow 0}\|\varepsilon f\|_{0}=0\right\}= \\
& =\left\{f \in \mathbf{R}^{X} \mid \lim _{t \rightarrow \infty} \mu^{*}\{|f|>t\}=0\right\}
\end{aligned}
$$

whenever $f$ belongs to $\mathbf{R}^{X}$. Note that $\|\cdot\|_{0}$-convergence in $\mathbf{R}^{X}$ is actually convergence in $\mu^{*}$-measure, that is $\left\|f_{n}-f\right\|_{0} \rightarrow 0$, if and only if $\mu^{*}\left\{\left\|f_{n}-f\right\|>\varepsilon\right\} \rightarrow 0$ as $n \rightarrow \infty$, for all $\varepsilon>0$ with $f_{n}$ and $f$ in $\mathbf{R}^{X}$ for $n \geq 1$. It is well-known that we have:

$$
\left(L^{0}\left(\mu^{*}\right),\|\cdot\|_{0}\right) \text { is a Fréchet space. }
$$

Let $M(\mu)$ denote the set of all $\mu$-measurable functions from $X$ into $\mathbf{R}$, then we define $L^{\tau_{\varphi}}(\mu)=L^{\tau_{\varphi}}\left(\mu^{*}\right) \cap M(\mu), \Lambda^{\sigma_{\varphi}}(\mu)=\Lambda^{\sigma_{\varphi}}\left(\mu^{*}\right) \cap M(\mu)$ and $L^{0}(\mu)=L^{0}\left(\mu^{*}\right) \cap$ $M(\mu)$. It is well-known that we have, see [11]:

(2.4) If $\lim _{t \rightarrow \infty} \varphi(t)=\infty$, then $L^{\tau_{\varphi}}\left(\mu^{*}\right) \subset L^{0}\left(\mu^{*}\right)$ and the natural injection is continuous. The space $L^{\tau_{\varphi}}(\mu)$ is a closed linear subspace of $L^{\tau_{\varphi}}\left(\mu^{*}\right)$. If $\varphi$ is moreover convex, then $L^{\tau_{\varphi}}(\mu)$ is a Banach space relative to the Orlicz norm $\|\cdot\|_{\tau_{\varphi}}$

In exactly the same way one might prove:

(2.5) If $\lim _{t \rightarrow \infty} \varphi(t)=\infty$, then $\Lambda^{\sigma_{\varphi}}\left(\mu^{*}\right) \subset L^{0}\left(\mu^{*}\right)$ and the natural injection is continuous. The space $\Lambda^{\sigma_{\varphi}}(\mu)$ is a closed linear subspace of $\Lambda^{\sigma_{\varphi}}\left(\mu^{*}\right)$. If $\varphi$ is moreover convex at 0 , i.e. $\varphi(\lambda t) \leq \lambda \varphi(t)$ for all $\lambda \in[0,1]$ and all $t \geq 0$, then $\Lambda^{\sigma_{\varphi}}(\mu)$ is a Fréchet space relative to the norm $\|\cdot\|_{\sigma_{\varphi}}^{1 / 2}$.

As another point we evidently have:

(2.6) $L^{0}(\mu)$ is a closed linear subspace of $L^{0}\left(\mu^{*}\right)$ and thus a Fréchet space relative to the norm $\|\cdot\|_{0}$.

According to the fact that the Orlicz norm $\|\cdot\|_{\tau_{\varphi}}$ and the Laurent norm $\|\cdot\|_{\sigma_{\varphi}}$ are induced by the same function $\varphi$ we will say that $\|\cdot\|_{\tau_{\varphi}}$ and $\|\cdot\|_{\sigma_{\varphi}}$ are associated each to other. The following elementary inequality establishes a basic connection between the associated Orlicz and Laurent norms and is occasionally useful: 


$$
\|f\|_{\sigma_{\varphi}} \leq\|f\|_{\tau_{\varphi}}
$$

being valid for all $f \in \mathbf{R}^{X}$. Let us now pass to concrete examples. First take for above $\varphi$ the function $\varphi_{p}(t)=t^{p}$ for $t \geq 0$ with $1 \leq p<\infty$. Then we evidently have:

$$
\begin{aligned}
& \|f\|_{\tau_{\varphi}}=\left(\int^{*}|f|^{p} d \mu\right)^{1 / p} \\
& \|f\|_{\sigma_{\varphi}}=\sup _{t>0}\left(t^{p} \cdot \mu^{*}\{|f|>t\}\right)^{1 / p}
\end{aligned}
$$

for all $f \in \mathbf{R}^{X}$. The norm appeared in (2.8) is the classical $L^{p}$-norm $\|\cdot\|_{p}$, while the norm appeared in (2.9) is often called the weak $L^{p}$-norm and is usually denoted by $\|\cdot\|_{p, \infty}$. Let us say that the given weak $L^{p}$-norms take a significant place in the study of various Kahane-Khintchine inequalities in Orlicz spaces, see [10], [11] and [12]. According to (2.7) we have:

$$
\|f\|_{p, \infty} \leq\|f\|_{p}
$$

for all $f \in \mathbf{R}^{X}$ and all $1 \leq p<\infty$. However it is easily verified that an $L^{p}$-norm and the associated weak $L^{p}$-norm are not equivalent in general. Now take for above $\varphi$ the function $\phi_{p}(t)=\exp \left(t^{p}\right)-1$ for $t \geq 0$ and $1 \leq p<\infty$. Then it is easy to see that the explicit forms of the norms are:

$$
\begin{aligned}
& \|f\|_{\tau_{\psi_{p}}}=\inf \left\{a>0 \mid \int^{*} \phi_{p}\left(a^{-1}|f|\right) d \mu \leq 1\right\} \\
& \|f\|_{\sigma_{\psi_{p}}}=\sup _{\lambda>0}\left(\lambda^{p} / \log \left(1+\frac{1}{\mu^{*}\{|f|>\lambda\}}\right)\right)^{1 / p}
\end{aligned}
$$

for all $f \in \mathbf{R}^{X}$. The norm appeared in (2.11) is the classical Orlicz norm $\|\cdot\|_{\psi_{p}}$ induced by $\phi_{p}$, and the norm appeared in (2.12) is the associated Laurent norm which will be in next denoted by $\|\cdot\|_{\phi_{p}, \infty}$. According to (2.7) we have:

$$
\|f\|_{\varphi_{p}, \infty} \leq\|f\|_{\psi_{p}}
$$

for all $f \in \mathbf{R}^{X}$ and all $1 \leq p<\infty$. However in this case we have even more. Namely there exists a constant $C_{p}>0$ such that:

$$
\|f\|_{\psi_{p}} \leq C_{p} \cdot\|f\|_{\psi_{p}, \infty}
$$

for all $f \in \mathbf{R}^{X}$ and all $1 \leq p<\infty$. This fact is easily established and we shall leave its verification to the reader. However the given observation is significant since it shows that the essence of a continuity principle for operators in exponen- 
tial type Orlicz spaces differs from the known ones in $L^{p}$-spaces as it was explained in introduction. We proceed by considering the measure-theoretic setting of Stein-Sawyer's continuity principles in some more details. Let $(X, \mathscr{A}, \mu)$ be a finite measure space, then a map $E$ from $X$ into itself is called an endomorphism, if $E$ is measurable and satisfies $\mu \circ E^{-1}=\mu$. Endomorphisms are often called measure-preserving transformations. Let $E$ be an endomorphism in $(X, \mathscr{A}, \mu)$, then a set $A$ in $\mathscr{A}$ is said to be $E$-invariant, if $E^{-1}(A)=A$. The family $\mathscr{A}_{E}$ of all $E$-invariant sets in $\mathscr{A}$ is a $\sigma$-algebra in $X$. The endomorphism $E$ is called ergodic, if $\mu(A) \cdot \mu\left(A^{c}\right)=0$ for all $A \in \mathscr{A}_{E}$, while $E$ is called (strongly) mixing, if we have:

$$
\lim _{i \rightarrow \infty} \mu(X) \cdot \mu\left(A \cap E^{-i}(B)\right)=\mu(A) \cdot \mu(B)
$$

for all $A, B \in \mathscr{A}$. If $E$ is mixing, then it is obviously ergodic. It is well-known that mixing endomorphisms arise naturally in many concrete examples, while ergodic endomorphisms take a central place in the study of various ergodic theorems having the origin and interpretation in statistical mechanics. Therefore mixing condition (1.3) imposed on a family of measure-preserving transformations $\mathscr{E}$ in $(X, \mathscr{A}, \mu)$ becomes natural and acceptable. Let us for instance point out a trivial fact that if $E$ is a mixing endomorphism in $(X, \mathscr{A}, \mu)$, then the family of measure-preserving transformations $\mathscr{E}=\left\{E^{i} \mid i \geq 1\right\}$ is mixing in the sense of (1.3). Moreover one can easily verify that we have:

(2.15) If $E$ is an ergodic endomorphism in $(X, \mathscr{A}, \mu)$, then the family of measure-preserving transformations $\mathscr{E}=\left\{E^{\imath} \mid i \geq 1\right\}$ is mixing in the sense of (1.3).

A similar interpretation of commuting condition (1.4) is possible, see [5] (p.7). To conclude this section let us point out an important consequence of mixing condition (1.3) which can be easily deduced by induction using the inequality $1-x$ $\leq \exp (-x)$ for $x \in \mathbf{R}$, see [5] (p.10):

(2.16) If $\mathscr{E}$ is a family of measure-preserving transformations in $(X, \mathscr{A}, \mu)$ which is mixing in the sense of (1.3), then for any $A \in \mathscr{A}$ with $\mu(A)>0$ and every $n \geq 1$, there exist $E_{1}, \ldots, E_{n} \in \mathscr{E}$ such that:

$$
\mu\left(E_{1}^{-1}(A) \cup \ldots \cup E_{n}^{-1}(A)\right) \geq \frac{1}{2} \cdot \mu(X)
$$

provided that $n \cdot \mu(A) \geq \mu(X)$. 


\section{The continuity principle in exponential type Orlicz spaces}

In this section we present the basic results of the paper. Let $(X, \mathscr{A}, \mu)$ be a finite measure space, and let $M(\mu)$ denote the linear space of all $\mu$-measurable functions from $X$ into $\mathbf{R}$. Let $\phi_{p}(t)=\exp \left(t^{p}\right)-1$ for $t \geq 0$ and $1 \leq p<\infty$, and let $L^{\psi_{p}}(\mu)$ denote the Orlicz space with the Orlicz norm $\|\cdot\|_{\psi_{p}}$ induced by $\phi_{p}$ for 1 $\leq p<\infty$. Given a sequence of linear operators $\left\{T_{n} \mid n \geq 1\right\}$ from $L^{\psi_{p}}(\mu)$ into $M(\mu)$, we recall that $T^{*} f=\sup _{n \geq 1}\left|T_{n} f\right|$ for $f \in L^{\psi_{p}}(\mu)$ with $1 \leq p<\infty$. Then we have:

Theorem 3.1. Let $(X, \mathscr{A}, \mu)$ be a finite measure space, and let $\left\{T_{n} \mid n \geq 1\right\}$ be a sequence of linear operators from $L^{\psi_{p}}(\mu)$ into $M(\mu)$ with $1 \leq p<\infty$. Suppose moreover that there exists a family $\mathscr{E}$ of measure-preserving transformations in $(X, \mathscr{A}, \mu)$ such that the three conditions are satisfied:

(3.1) Every $T_{n}$ is positive and continuous in $\mu$-measure for $n \geq 1$

(3.2) $\mathscr{E}$ is mixing in the sense of (1.3)

(3.3) $\left\{T_{n} \mid n \geq 1\right\}$ and $\mathscr{E}$ commute in the sense of (1.4).

Then $T^{*} f<\infty \mu$-a.e. for all $f \in L^{\phi_{p}}(\mu)$, if and only if the following inequality is satisfied:

$$
\left\|T^{*} f\right\|_{\psi_{p}} \leq C_{p} \cdot\|f\|_{\psi_{p}}
$$

for all $f \in L^{\psi_{p}}(\mu)$ with some $C_{p}>0$ and for given $1 \leq p<\infty$.

Proof. It is no restriction to assume that $\mu$ is a probability measure, that is $\mu(X)=1$. Furthermore note that if (3.4) is satisfied, then evidently $T^{*} f<\infty$ $\mu$-a.e. for $f \in L^{\phi_{p}}(\mu)$ with $1 \leq p<\infty$. Therefore to complete the proof it is enough to show the reverse implication. We begin in this direction by establishing the following inequality:

$$
\left\|\max _{1 \leq j \leq n}\left|f_{j}\right|\right\|_{\psi_{p}} \leq(\Delta \cdot \log n)^{1 / p} \cdot \max _{1 \leq j \leq n}\left\|f_{j}\right\|_{\psi_{p}}
$$

being valid for all $f_{1}, \ldots, f_{n} \in L^{\psi_{p}}(\mu)$ with $n \geq 2$ and $\Delta=2 / \log 2$, where $1 \leq p$ $<\infty$ is given and fixed. To prove (3.5) it is no restriction to assume that $\left\|f_{j}\right\|_{\psi_{p}}$ $\leq 1$ for $j=1, \ldots, n$. Since $\Delta \cdot \log n \geq 1$, then by Jensen's inequality we find:

$$
\int \exp \left(\frac{1}{(\Delta \cdot \log n)^{1 / p}} \cdot \max _{1 \leq s \leq n}|f|\right)^{p} d \mu=
$$




$$
\begin{aligned}
& =\int \exp \left(\frac{1}{\Delta \cdot \log n} \cdot \max _{1 \leq j \leq n}\left|f_{j}\right|^{p}\right) d \mu \\
& \leq\left(\int \exp \left(\max _{1 \leq j \leq n}\left|f_{j}\right|^{p}\right) d \mu\right)^{1 /(\Delta \log n)} \\
& \leq(2 n)^{1 /(\Delta \log n)}=\exp \left(\frac{\log (2 n)}{2 \log n} \cdot \log 2\right) \leq 2 .
\end{aligned}
$$

Hence (3.5) follows directly by the definition of the Orlicz norm $\|\cdot\|_{\varphi_{p}}$. Let us further proceed by considering a map $F$ from $X$ into $\mathbf{R}$ defined by:

$$
F(x)=\frac{1}{(\Delta \cdot \log n)^{1 / p}} \cdot \max _{1 \leq \jmath \leq n} f_{j}(x)
$$

for $x \in X$ and $n \geq 1$, where $f \geq 0$ in $L^{\phi_{p}}(\mu)$ with $\|f\|_{\psi_{p}}=1$ is given, and $f_{j}=$ $f \circ E_{j}$ with some $E_{j} \in \mathscr{E}$ for $j=1, \ldots, n$ which will be introduced precisely later on. Let us however notice that according to (3.5) we have:

$$
\|F\|_{\phi_{p}} \leq 1
$$

for any choice of $E_{j}$ 's in $\mathscr{E}$. By positivity of $T_{m}$ we find:

$$
T_{m} F \geq \frac{1}{(\Delta \cdot \log n)^{1 / p}} \cdot T_{m} f_{j}
$$

for all $1 \leq j \leq n$ and all $m \geq 1$, and therefore we have:

$$
T^{*} F \geq \frac{1}{(\Delta \cdot \log n)^{1 / p}} \cdot \max _{1 \leq j \leq n} T^{*} f_{j} .
$$

Let us now for given $\lambda>0$ put $A=\left\{T^{*} f>\lambda\right\}$, and let $A_{j}=E_{j}^{-1}(A)$ for $j=$ $1, \ldots, n$. Then by (3.3) and (3.6) we may easily conclude:

$$
\mu\left\{T^{*} F>\frac{\lambda}{(\Delta \cdot \log n)^{1 / p}}\right\} \geq \mu\left\{\max _{1 \leq j \leq n} T^{*} f_{j}>\lambda\right\} \geq \mu\left(\cup_{j=1}^{n} A_{j}\right) .
$$

According to (2.16) we can choose $E_{1}, \ldots, E_{n} \in \mathscr{E}$ in such a way that $\mu\left(\cup_{j=1}^{n} A_{j}\right) \geq 1 / 2$ in the case where we have:

$$
n \cdot \mu(A) \geq 1 \text {. }
$$

Assuming (3.8) then by (3.7) we get:

$$
\mu\left\{T^{*} F>\frac{\lambda}{(\Delta \cdot \log n)^{1 / p}}\right\} \geq 1 / 2 .
$$


However every $T_{m}$ is by assumption (3.1) continuous in $\mu$-measure. Therefore Banach's priciple (1.1) may be applied on the function $F$. Since $\|F\|_{\psi_{p}} \leq 1$, in this way we can find $C>0$ large enough to satisfy:

$$
\mu\left\{T^{*} F>C\right\}<1 / 2 .
$$

So putting $\lambda=C \cdot(\Delta \cdot \log n)^{1 / p}$ in (3.9) we obtain a contradiction with (3.10). Thus (3.8) is false. In other words we may conclude:

$$
\mu\left\{T^{*} f>C \cdot(\Delta \cdot \log n)^{1 / p}\right\}<1 / n
$$

for all $n \geq 2$. From this inequality we may easily deduce the following inequality:

$$
\mu\left\{T^{*} f>\lambda \cdot\|f\|_{\psi_{p}}\right\} \leq C_{1} \cdot \exp \left(-C_{2} \cdot \lambda^{p}\right)
$$

being valid for all $\lambda>0$ and all $f \in L^{\psi_{p}}(\mu)$ with some numerical constants $C_{1}, C_{2}$ $>0$. Hence we may easily obtain:

$$
\begin{aligned}
& \int\left(\exp \left(\frac{T^{*} f}{\gamma \cdot\|f\|_{\psi_{p}}}\right)^{p}-1\right) d \mu= \\
& \quad=\int_{0}^{\infty} \mu\left\{\exp \left(\frac{T^{*} f}{\gamma \cdot\|f\|_{\psi_{p}}}\right)^{p}-1>t\right\} d t \\
& \quad \leq \int_{0}^{\infty} C_{1} \cdot(1+t)^{-\gamma \cdot C_{2}} d t
\end{aligned}
$$

for all $\gamma>0$ and all $f \in L^{\psi_{p}}(\mu)$. Since the right side above obviouly tends to zero when $\gamma$ tends to infinity, we may conclude that for some numerical constant $C_{p}>0$ the following inequality is satisfied:

$$
\left\|T^{*} f\right\|_{\phi_{p}} \leq C_{p} \cdot\|f\|_{\phi_{p}}
$$

for all $f \in L^{\psi_{p}}(\mu)$. This fact completes the proof of the theorem.

In order to formulate an immediate consequence of the preceding result let us recall that $\|\cdot\|_{\psi_{p}, \infty}$ denotes the Laurent norm given by (2.12) for $1 \leq p<\infty$. Then we have:

COROLlaRY 3.2. Under the hypotheses in Theorem 3.1 we have $T^{*} f<\infty$ $\mu$-a.e. for all $f \in L^{\psi_{p}}(\mu)$, if and only if the following inequality is satisfied:

$$
\left\|T^{*} f\right\|_{\psi_{p} \infty} \leq C_{p} \cdot\|f\|_{\psi_{p}}
$$

for all $f \in L^{\psi_{p}}(\mu)$ with some $C_{p}>0$ and for given $1 \leq p<\infty$. In particular, if 
$T^{*} f<\infty \mu$-a.e. for all $f \in L^{\psi_{p}}(\mu)$, then we have:

$$
\mu\left\{T^{*} f>\lambda \cdot\|f\|_{\psi_{p}}\right\} \leq C_{p} \cdot \exp \left(-D_{p} \cdot \lambda^{p}\right)
$$

for all $\lambda>0$ and all $f \in L^{\psi_{p}}(\mu)$ with some $C_{p}, D_{p}>0$ and for given $1 \leq p<\infty$.

Proof. Straightforward by Theorem 3.1, (2.13) and (2.14).

This result establishes a continuity principle for positive linear operators in exponential type Orlicz spaces under rather weak hypotheses. It is however interesting to observe that the main inequality in the statement of the continuity principle, that is inequality (3.4) in Theorem 3.1, may be interpreted as a dominated ergodic theorem in the given exponential type Orlicz spaces, at least when the linear operators $T_{n}$ have the average form given by:

$$
T_{n} f=\frac{1}{n} \sum_{j=0}^{n-1} T^{j} f
$$

for some linear operator $T$ in $L^{1}(\mu)$ with $f \in L^{1}(\mu)$ and $n \geq 1$. However we shall now see that in the setting of the well-known dominated ergodic theorems in $L^{p}$-spaces these theorems contain enough informations to provide the eatablishment of inequality (3.4) in Theorem 3.1 in a rather elementary way. Let $(X, \mathscr{A}, \mu)$ be a finite measure space, let $M(\mu)$ denote the linear space of all $\mu$-measurable functions from $X$ into $\mathbf{R}$, and let $T$ be a linear operator from $L^{p}(\mu)$ into $M(\mu)$ for some $1 \leq p<\infty$. Then $T$ is said to be a contraction in $L^{p}(\mu)$, if we have $\|T f\|_{p}$ $\leq\|f\|_{p}$ for all $f \in L^{p}(\mu)$. And $T$ is said to be an $L^{1}-L^{\infty}$-contraction, if $T$ is a contraction in $L^{1}(\mu)$ and $\|T f\|_{\infty} \leq 1$ whenever $\|f\|_{\infty} \leq 1$. Having $T$ we may consider a sequence of linear operators $\left\{T_{n} \mid n \geq 1\right\}$ in $L^{p}(\mu)$ defined by (3.12) for all $f \in$ $L^{p}(\mu)$. As usual in this case we put $T^{*} f=\sup _{n \geq 1}\left|T_{n} f\right|$ for all $f \in L^{p}(\mu)$. Let $1<p<\infty$ be given and fixed, then it is well-known if any of the following three conditions is fulfilled:

(3.13) (Wiener's dominated ergodic theorem)

$T^{\jmath} f=f \circ \tau^{j}$ for $j=1,2 \ldots$, where $\tau$ is an endomorphism in $(X, \mathscr{A}, \mu)$

(3.14) (Dunford-Schwartz's dominated ergodic theorem)

$T$ is an $L^{1}-L^{\infty}$-contraction

(3.15) (Akcoglu's dominated ergodic theorem)

$T$ is a positive contraction in $L^{p}(\mu)$

then the following inequality is satisfied: 


$$
\left\|T^{*} f\right\|_{p} \leq \frac{p}{p-1} \cdot\|f\|_{p}
$$

for all $f \in L^{p}(\mu)$, see [9]. Moreover in the case where (3.13) or (3.14) is satisfied we have:

$$
\left\|T^{*} f\right\|_{1, \infty} \leq\|f\|_{1}
$$

for all $f \in L^{1}(\mu)$, see [9]. It turns out that the constants appearing in inequality (3.16) are sufficiently good to provide its extension to exponential type Orlicz spaces. In order to clarify some technical details in the proof of this fact we shall recall that we have:

$$
\varepsilon_{p, q} \cdot\|f\|_{p} \leq\left\|f_{\psi_{q}} \leq \delta_{q, \infty} \cdot\right\| f \|_{\infty}
$$

for all $f \in M(\mu)$ and all $1 \leq p, q<\infty$ with some numerical constants $\varepsilon_{p, q}, \delta_{q, \infty}>$ 0 . In particular we may deduce:

$$
L^{\infty}(\mu) \subset L^{\psi_{q}}(\mu) \subset \cap_{p>1} L^{\mathrm{p}}(\mu) \subset L^{1}(\mu)
$$

for all $1 \leq q<\infty$. A dominated ergodic theorem in exponential type Orlicz spaces may be now stated as follows.

Theorem 3.3. Let $(X, \mathscr{A}, \mu)$ be a finite measure space, and let $T$ be a linear operator satisfying any of above conditions (3.13), (3.14) and (3.15). Let us consider a sequence of linear operators $\left\{T_{n} \mid n \geq 1\right\}$ defined by (3.12) for all $f \in L^{\phi_{p}}(\mu)$ with some $1 \leq p<\infty$, and let us put $T^{*} f=\sup _{n \geq 1}\left|T_{n} f\right|$ for $f \in L^{\psi_{p}}(\mu)$. Then the following inequality is satisfied:

$$
\left\|T^{*} f\right\|_{\psi_{p}} \leq C_{p} \cdot\|f\|_{\psi_{p}}
$$

for all $f \in L^{\phi_{p}}(\mu)$ with some $C_{p}>0$ and for given $1 \leq p<\infty$. Moreover we have:

$$
\mu\left\{T^{*} f>\lambda \cdot\|f\|_{\psi_{p}}\right\} \leq C_{p} \cdot \exp \left(-D_{p} \cdot \lambda^{p}\right)
$$

for all $\lambda>0$ and all $f \in L^{\phi_{p}}(\mu)$ with some $C_{p}, D_{p}>0$ and for given $1 \leq p<\infty$.

Proof. It is no restriction to assume that $\mu$ is a probability measure, that is $\mu(X)=1$. First suppose that $p>1$, then by (3.16) we have:

$$
\begin{aligned}
\int \exp & \left(\frac{T^{*} f}{C \cdot\|f\|_{\phi_{p}}}\right)^{p} d \mu= \\
& =\sum_{k=0}^{\infty} \frac{1}{k !} \cdot \frac{1}{C^{k p} \cdot\|f\|_{\psi_{p}}^{k p}} \cdot \int\left|T^{*} f\right|^{k p} d \mu
\end{aligned}
$$




$$
\begin{aligned}
& \leq \sum_{k=0}^{\infty} \frac{1}{k !} \cdot \frac{1}{C^{k p} \cdot\|f\|_{\phi_{p}}^{k p}} \cdot\left(\frac{k p}{k p-1}\right)^{k p} \cdot \int|f|^{k p} d \mu \\
& \leq K_{p} \cdot \int\left(\sum_{k=0}^{\infty} \frac{1}{k !} \cdot \frac{1}{C^{k p} \cdot\|f\|_{\phi_{p}}^{k p}} \cdot|f|^{k p}\right) d \mu \\
& =K_{p} \cdot \int \exp \left(\frac{|f|}{C \cdot\|f\|_{\psi_{p}}}\right)^{p} d \mu \leq 2
\end{aligned}
$$

for some $K_{p}>0$ and $C:=C_{p}>0$ sufficiently large. Hence (3.20) follows directly by the definition of the Orlicz norm $\|\cdot\|_{\psi_{p}}$. Now suppose that $p=1$, then by (3.16) we have:

$$
\begin{aligned}
& \int \exp \left(\frac{T^{*} f}{C \cdot\|f\|_{\psi_{p}}}\right) d \mu=\sum_{k=0}^{\infty} \frac{1}{k !} \cdot \frac{1}{C^{k} \cdot\|f\|_{\phi_{1}}^{k}} \cdot \int\left|T^{*} f\right|^{k} d \mu \leq \\
& \leq 1+\frac{1}{C \cdot\|f\|_{\phi_{1}}} \cdot \int\left|T^{*} f\right| d \mu+\sum_{k=2}^{\infty} \frac{1}{k !} \cdot \frac{1}{C^{k} \cdot\|f\|_{\phi_{1}}^{k}} \cdot\left(\frac{k}{k-1}\right)^{k} \cdot \int|f|^{k} d \mu \\
& \leq \frac{1}{C \cdot\|f\|_{\psi_{1}}} \cdot \int\left|T^{*} f\right| d \mu+K_{1} \cdot \sum_{k=0}^{\infty} \frac{1}{k !} \cdot \frac{1}{C^{k} \cdot\|f\|_{\phi_{1}}^{k}} \cdot \int|f|^{k} d \mu
\end{aligned}
$$

for all $C>0$ with some $K_{1}>0$. Hence by Jensen's inequality, (3.16) and (3.18) we get:

$$
\int \exp \left(\frac{T^{*} f}{C \cdot\|f\|_{\psi_{1}}}\right) d \mu \leq \frac{2}{C \cdot \varepsilon_{2,1}}+K_{1} \cdot \int \exp \left(\frac{|f|}{C \cdot\|f\|_{\psi_{1}}}\right) d \mu \leq 2
$$

for $C:=C_{1}>0$ sufficiently large. Hence (3.20) follows directly by the definition of the Orlicz norm $\|\cdot\|_{\psi_{1}}$. Inequality (3.21) follows straightforward by (3.20) and (2.13).

Let us in addition say that a part of the preceding theorem was already recorded and used earlier in an equivalent formulation in [16]. This result has found also an application in [1]. In this context and due to the fact that the given exponential Orlicz norms are equivalent to the associated Laurent norms we may conclude that the continuity priciple established in Theorem 3.1 simultaneously forms a dominated ergodic theorem for more general positive linear operators than those considered in Theorem 3.3. Actually it is instructive to observe that the average form of the operators from Theorem 3.3 is compensated by the mixing-commuting condition imposed on the phase space in Teorem 3.1. The next example shows that these facts may in a quite natural setting be the only available 
ones and at the same time serves an application of the continuity principle of Theorem 3.1 itself.

Example 3.4. Let $\xi=\left\{\xi_{j} \mid j \geq 1\right\}$ be a stationary ergodic sequence of real valued random variables defined on a probability space $(\Omega, \mathscr{F}, P)$, and let $\mathscr{G}_{n}=$ $\sigma\left(\xi_{n}, \xi_{n+1} \ldots\right)$ denote the $\sigma$-algebra generated by $\xi_{n}, \xi_{n+1} \ldots$ for $n \geq 1$. Let us define:

$$
T_{n} f=E\left[f \mid \mathscr{G}_{n}\right]
$$

for all $f \in L^{1}(P)$ and all $n \geq 1$. Then $\left\{T_{n} \mid n \geq 1\right\}$ is a sequence of positive linear operators from $L^{1}(P)$ into itself. Moreover every $T_{n}$ is obviously continuous in $P$-measure in every $L^{p}(P)$, and in every $L^{\psi_{p}}(P)$ as well, for $1 \leq p<\infty$. So (3.1) in Theorem 3.1 is satisfied. In what follows it is no restriction to assume that our probability space $(\Omega, \mathscr{F}, P)$ equals $\left(\mathbf{R}^{\mathrm{N}}, \mathscr{B}\left(\mathbf{R}^{\mathrm{N}}\right), P\right)$ with the unilateral shift $\theta$ as an ergodic endomorphism. Therefore by $(2.15)$ the family of measure-preserving transformations $\mathscr{E}=\left\{\theta^{i} \mid i \geq 1\right\}$ is mixing in the sense of (1.3). So (3.2) in Theorem 3.1 is also satisfied. Moreover one can easily verify that we have:

$$
\left(T_{n} f\right) \circ \theta=T_{n+1}(f \circ \theta)
$$

for all $f \in L^{1}(P)$ and all $n \geq 1$. Hence by induction we get:

$$
\left(T_{n} f\right) \circ \theta^{i}=T_{n+i}\left(f \circ \theta^{i}\right)
$$

for all $f \in L^{1}(\mu)$ and all $n, i \geq 1$. Putting $T^{*} f=\sup _{n \geq 1}\left|T_{n} f\right|$ for $f \in L^{1}(P)$ we may easily conclude:

$$
\left(T^{*} f\right) \circ \theta^{i} \leq T^{*}\left(f \circ \theta^{i}\right)
$$

for all $f \in L^{1}(P)$ and all $i \geq 1$. In other words the sequence $\left\{T_{n} \mid n \geq 1\right\}$ and the family $\mathscr{E}$ commute in the sense of (1.4). Thus (3.3) in Theorem 3.1 is satisfied, as well as the hypotheses in Sawyer's continuity principle (1.6). Moreover by applying reversed martingale convergence theorem we know that $T_{n} f$ converges $P$-a.e. as $n \rightarrow \infty$ for every $f \in L^{1}(P)$. Therefore $T^{*} f<\infty P$-a.e. for all $f \in$ $L^{1}(P)$, and by Theorem 3.1 we may establish the following inequality:

$$
\left\|\sup _{n \geq 1}\left|E\left[f \mid \mathscr{G}_{n}\right]\right|\right\|_{\phi_{p}} \leq C_{p} \cdot\|f\|_{\phi_{p}}
$$

being valid for all $f \in L^{\psi_{p}}(P)$ with some $C_{p}>0$ and for given $1 \leq p<\infty$. Moreover by (2.13) hence we may deduce:

$$
P\left\{\sup _{n \geq 1}\left|E\left[f \mid \mathscr{G}_{n}\right]\right|>\lambda \cdot\|f\|_{\psi_{p}}\right\} \leq C_{p} \cdot \exp \left(-D_{p} \cdot \lambda^{p}\right)
$$


for all $\lambda>0$ and all $f \in L^{\psi_{p}}(P)$ with some $C_{p}, D_{p}>0$ and for given $1 \leq p<\infty$. By applying Sawyer's continuity principle (2.6) we may establish the following inequality:

$$
P\left\{\sup _{n \geq 1}\left|E\left[f \mid \mathscr{G}_{n}\right]\right|>\lambda\right\} \leq \frac{C_{p}}{\lambda^{p}} \int|f|^{p} d P
$$

being valid for all $f \in L^{p}(P)$ with some $C_{p}>0$ and for given $1 \leq p<\infty$. In other words we can write:

$$
\left\|\sup _{n \geq 1}\left|E\left[f \mid \mathscr{G}_{n}\right]\right|\right\|_{p, \infty} \leq C_{p} \cdot\|f\|_{p}
$$

with $f, C_{p}$ and $p$ as above. However it is instructive to notice that the constants $C_{p}$ appearing in (3.24) and (3.25) are not explicitly determined, so a potential application of (3.24) and (3.25) in a direct proof of (3.22) and (3.23) as in the proof of Theorem 3.3 seems to be not available.

We conclude our investigation on the continuity priciple in this paper by considering the case where the operators $T_{n}$ are defined by means of a matrix summation method. It turns out that in this case a stronger version of Theorem 3.1 is valid. This fact is already observed in [17] in the $L^{p}$-setting, and we shall here prove its extension to exponential type Orlicz spaces. The key argument for this improvement relies upon the conjugacy lemma of Halmos, see [6] (p.77), and is similar to the one used for the establishment of the domination priciple of Conze, see [2] and [17]. In order to introduce the hypotheses we shall first recall some definitions and known facts which will be of use in the sequel. Let $(X, \mathscr{A}, \mu)$ be a finite measure space, then a measure-preserving transformation $\tau$ in $(X, \mathscr{A}, \mu)$ is said to be an automorphism in $(X, \mathscr{A}, \mu)$, if $\tau$ is bijective and $\tau^{-1}$ is measurepreserving. The family of all automorphisms in $(X, \mathscr{A}, \mu)$ will be denoted by $\mathscr{C}$. The family $\mathscr{C}$ forms a group with composition as the binary operation. If $\tau$ belongs to $\mathscr{C}$, then $\tau f=f \circ \tau$ for $f \in L^{2}(\mu)$ defines a unitary operator on $L^{2}(\mu)$. It is well-known that the strong and the weak topology restricted to the set of all unitary operators coincide. Therefore the specializations of these topologies to $\mathscr{C}$ are the same. The given topology is called the weak topology for $\mathscr{C}$, and we have that $\tau_{n} \rightarrow \tau$ in $\mathscr{C}$ if and only if any of the four equivalent conditions is satisfied:

$$
\begin{aligned}
& f \circ \tau_{n} \rightarrow f \circ \tau \text { in } L^{2}(\mu) \text { for all } f \in L^{2}(\mu) \\
& \mu\left(\tau_{n}^{-1}(A) \Delta \tau^{-1}(A)\right) \rightarrow 0 \text { for all } A \in \mathscr{A} \\
& \mu\left(\tau_{n}(A) \Delta \tau(A)\right) \rightarrow 0 \text { for all } A \in \mathscr{A}
\end{aligned}
$$




$$
f \circ \tau_{n} \rightarrow f \circ \tau \text { in } L^{p}(\mu) \text { for all } f \in L^{p}(\mu)
$$

where $1 \leq p<\infty$ is given and fixed. Moreover with respect to this topology the group $\mathscr{C}$ becomes a topological group, see [6] (p.62). In the sequel we will be working under the hypothesis that the measure space $(X, \mathscr{A}, \mu)$ is isomorphic (pointwise) to the unit interval $[0,1]$ with Lebesgue measure. In this case we will say that $(X, \mathscr{A}, \mu)$ is a Lebesgue space. It is well-known for instance, that every polish space $X$ (with $\mathscr{A}$ being the completion of the Borel $\sigma$-algebra $\mathscr{B}(X)$ with respect to any probability measure $\mu$ on $\mathscr{B}(X)$ ) is a Lebesgue space, see [13] (p.16-17). It turns out that this hypothesis is sufficiently regular to provide fruitful facts on the weak topology of the group of automorphisms, see [6] (p.61). For instance under this hypothesis the weak topology of $\mathscr{C}$ becomes metrizable and satisfies the first countability axiom, see [6] (p.63-64). Moreover recall that $\tau \in \mathscr{C}$ is said to be aperiodic, if $\mu\left\{x \in X \mid \tau^{n} x=x\right\}=0$ for all $n \geq 1$. Then we have, see [6] (p.77):

(3.30) (The conjugacy lemma)

If $\sigma \in \mathscr{C}$ is aperiodic, then the conjugate class $c(\sigma)=\left\{\tau^{-1} \sigma \tau \mid \tau \in \mathscr{C}\right\}$ of $\sigma$ is dense in $\mathscr{C}$.

It is easily verified that every ergodic automorphism in $(X, \mathscr{A}, \mu)$ is aperiodic. However one can easily establish that this fact might not be true for some other measure spaces. We turn to define the operators $T_{n}$ by means of a matrix summation method. For this suppose that an infinite matrix of real numbers $A=\left(a_{n k} \mid n\right.$, $k \geq 1$ ) and $1 \leq p<\infty$ are given and fixed. Let $\tau$ be from $\mathscr{C}$, then we may formally put:

$$
T_{n}^{\tau} f=\sum_{k=1}^{\infty} a_{n k}\left(f \circ \tau^{k}\right)
$$

for all $f \in L^{\psi_{p}}(\mu)$ and all $n \geq 1$. We will assume that the row vectors $a_{n}=\left(a_{n k} \mid\right.$ $k \geq 1$ ) belong to $l_{1}$. From this fact we may easily conclude that by (3.31) a continuous linear operator $T_{n}^{\tau}$ on $L^{\psi_{p}}(\mu)$ is defined for $n \geq 1$. Clarify that $T_{n}^{\tau} f$ is the $L^{\psi_{p}}$-limit of $\sum_{k=1}^{N} a_{n k}\left(f \circ \tau^{k}\right)$ when $N \rightarrow \infty$, for all $f \in L^{\psi_{p}}(\mu)$ and all $n \geq 1$. Moreover by (2.4) we may easily verify that every $T_{n}^{\tau}$ is continuous in $\mu$-measure for $n \geq 1$. Further we will assume that $a_{n k} \geq 0$ for all $n, k \geq 1$. Under this hypothesis every $T_{n}^{\tau}$ becomes positive for $n \geq 1$. As usual we shall denote:

$$
T_{\tau}^{*} f=\sup _{n \geq 1}\left|T_{n}^{\tau} f\right|
$$

for all $f \in L^{\psi_{p}}(\mu)$. It is instructive to notice that for any $\sigma \in \mathscr{C}$ we have: 


$$
\sigma\left(T_{\tau}^{*} f\right)=T_{\tau}^{*}(\sigma f)
$$

for all $f \in L^{\phi_{p}}(\mu)$ provided that $\sigma^{\circ} \tau=\tau^{\circ} \sigma$. In particular we find:

$$
\tau^{i}\left(T_{\tau}^{*} f\right)=T_{\tau}^{*}\left(\tau^{i} f\right)
$$

for all $f \in L^{\phi_{p}}(\mu)$ and all $i \geq 1$. In order to prove the main theorem under the above hypotheses we shall first state a lemma which is also of interest in itself.

LEMMA 3.5. Let $A=\left(a_{n k} \mid n, k \geq 1\right)$ be an infinite matrix of non-negative real numbers such that the row vectors $a_{n}=\left(a_{n k} \mid k \geq 1\right)$ belong to $l_{1}$ for all $n \geq 1$, and let $1 \leq p<\infty$ be given and fixed. Let $(X, \mathscr{A}, \mu)$ be a Lebesgue space, and let $T_{n}^{\tau}$ and $T_{\tau}^{*}$ be defined by means of $A$ as in (3.31) and (3.32) whenever $\tau \in \mathscr{C}$ and $n \geq 1$. Let $\mathscr{D}$ denote the set of all $\tau$ in $\mathscr{C}$ satisfying:

$$
\mu\left\{T_{\tau}^{*} f>C(\lambda)\right\} \leq D(\lambda)
$$

for all $\lambda>0$ and all $f \in L^{\phi_{p}}(\mu)$ with $\|f\|_{\psi_{p}}=1$, where $C$ and $D$ are given functions from ] $0, \infty$ [ into itself. Then $\mathscr{D}$ is closed in $\mathscr{C}$.

Proof. Suppose that $\tau_{p}$ belongs to $\mathscr{D}$ for all $p \geq 1$ and $\tau_{p} \rightarrow \tau$ in $\mathscr{C}$ as $p \rightarrow \infty$ for some $\tau \in \mathscr{C}$. Then it is enough to show the following inequality:

$$
\mu\left\{\sup _{1 \leq n \leq N}\left|\sum_{k=1}^{\infty} a_{n k}\left(f \circ \tau^{k}\right)\right|>C(\lambda)\right\} \leq D(\lambda)
$$

being valid for all $\lambda>0$, all $f \in L^{\phi_{p}}(\mu)$ with $\|f\|_{\psi_{p}}=1$, and all $N \geq 1$. Let $N \geq 1$ be given and fixed, and let $\varepsilon>0$ be given. Since $a_{n}$ by our hypothesis belongs to $l_{1}$ for all $n \geq 1$, there exists $M_{\varepsilon} \geq 1$ such that $\sum_{k=M_{\varepsilon}}^{\infty} a_{n k}<\varepsilon$ for all $1 \leq n$ $\leq N$. Therefore by (3.31), (2.4) and Markov's inequality we have:

$$
\begin{gathered}
\mu\left\{\sup _{1 \leq n \leq N}\left|\sum_{k=1}^{\infty} a_{n k}\left(f \circ \tau^{k}\right)\right|>C(\lambda)+\delta\right\} \leq \\
\mu\left\{\sup _{1 \leq n \leq N}\left|\sum_{k=1}^{P_{\varepsilon}} a_{n k}\left(f \circ \tau^{k}\right)\right|>C(\lambda)\right\}+\varepsilon \leq \\
\mu\left\{\sup _{1 \leq n \leq N}\left|\sum_{k=1}^{P_{\varepsilon}} a_{n k}\left(f \circ \tau_{p_{\varepsilon}}^{k}\right)\right|>C(\lambda)+\delta\right\}+2 \varepsilon \leq \\
\mu\left\{\sup _{1 \leq n \leq N}\left|\sum_{k=1}^{\infty} a_{n k}\left(f \circ \tau_{p_{\varepsilon}}^{k}\right)\right|>C(\lambda)\right\}+ \\
+\mu\left\{\sup _{1 \leq n \leq N}\left|\sum_{k=P_{\varepsilon}+1}^{\infty} a_{n k}\left(f \circ \tau_{p_{\varepsilon}}^{k}\right)\right|>\delta\right\}+2 \varepsilon \leq D(\lambda)+N \cdot \varepsilon \cdot \frac{1}{\delta} \cdot\|f\|_{1}+2 \varepsilon
\end{gathered}
$$


for all $\lambda, \delta>0$ and with some $P_{\varepsilon} \geq M_{\varepsilon}$ and $p_{\varepsilon} \geq 1$. Letting first $\varepsilon \downarrow 0$ and then $\delta \downarrow 0$ we get (3.35), and the proof is complete.

The improvement of Theorem 3.1 for the operators defined by matrix summation methods may be now stated as follows.

THEOREM 3.6. Let $A=\left(a_{n k} \mid n, k \geq 1\right)$ be an infinite matrix of non-negative real numbers such that the row vectors $a_{n}=\left(a_{n k} \mid k \geq 1\right)$ belong to $l_{1}$ for all $n \geq 1$, and let $1 \leq p<\infty$ be given and fixed. Let $(X, \mathscr{A}, \mu)$ be a Lebesgue space, and let $T_{n}^{\tau}$ and $T_{\tau}^{*}$ be defined by means of $A$ as in (3.31) and (3.32) whenever $\tau \in \mathscr{C}$ and $n \geq 1$. Then the following two statements are equivalent:

(3.36) There exists an ergodic automorphism $\sigma \in \mathscr{C}$ such that:

$$
T_{\sigma}^{*} f<\infty \quad \mu \text {-a.e. }
$$

$$
\text { for all } f \in L^{\varphi_{p}}(\mu)
$$

(3.37) The following inequality is satisfied:

$$
\sup _{\tau \in \mathscr{C}}\left\|T_{\tau}^{*} f\right\|_{\psi_{p}} \leq C_{p} \cdot\|f\|_{\psi_{p}}
$$

for all $f \in L^{\phi_{p}}(\mu)$ with some $C_{p}>0$.

Moreover in this case we have:

$$
\sup _{\tau \in \mathscr{C}} \mu\left\{T_{\tau}^{*} f>\lambda \cdot\|f\|_{\psi_{p}}\right\} \leq C_{p} \cdot \exp \left(-D_{p} \cdot \lambda^{p}\right)
$$

for all $\lambda>0$ and all $f \in L^{\psi_{p}}(\mu)$ with some $C_{p}, D_{p}>0$.

Proof. Since (3.37) implies (3.36) obviously, it is enough to show that (3.36) implies (3.37). For this put $\mathscr{C}=\left\{\sigma^{i} \mid i \geq 1\right\}$, then by (2.4), (2.15) and (3.33) we may easily verify that (3.1), (3.2) and (3.3) are satisfied. Thus by Theorem 3.1 we can find a constant $K_{p}>0$ such that:

$$
\left\|T_{\sigma}^{*} f\right\|_{\phi_{p}} \leq K_{p}
$$

for all $f \in L^{\psi_{p}}(\mu)$ with $\|f\|_{\psi_{p}}=1$. Let $c(\sigma)=\left\{\tau^{-1} \sigma \tau \mid \tau \in \mathscr{C}\right)$ be the conjugate class of $\sigma$. Let $\alpha=\tau^{-1} \sigma \tau$ be from $c(\sigma)$ for some $\tau \in \mathscr{C}$, then we clearly have:

$$
T_{n}^{\alpha}(f)=\tau\left(T_{n}^{\sigma}\left(\tau^{-1} f\right)\right)
$$

for all $f \in L^{\phi_{p}}(\mu)$. Hence we directly get:

$$
T_{\alpha}^{*}(f)=\tau\left(T_{\sigma}^{*}\left(\tau^{-1} f\right)\right)
$$


for all $f \in L^{\phi_{D}}(\mu)$. Thus by (3.39) we may conclude:

$$
\left\|T_{\alpha}^{*} f\right\|_{\psi_{p}} \leq K_{p}
$$

for all $f \in L^{\psi_{p}}(\mu)$ with $\|f\|_{\psi_{p}}=1$ and all $\alpha \in c(\sigma)$. Hence by (2.12) and (2.13) we easily find:

$$
\mu\left\{T_{\alpha}^{*} f>\lambda\right\} \leq\left(\exp \left(\lambda / K_{p}\right)^{p}-1\right)^{-1}
$$

being valid for all $\lambda>0$, all $f \in L^{\psi_{p}}(\mu)$ with $\|f\|_{\psi_{p}}=1$, and all $\alpha \in c(\sigma)$. By Lemma 3.5 the family of all $\alpha$ in $\mathscr{C}$ satisfying (3.41) is closed in $\mathscr{C}$. Therefore by the conjugacy lemma (3.30) we may conclude that (3.41) holds for every $\alpha$ in $\mathscr{C}$. Hence (3.40) follows straightforward by (2.12) and (2.14) for any $\alpha$ in $\mathscr{C}$, where $K_{p}$ should by replaced by a constant $L_{p} \geq K_{p}$ which is not depending on $\alpha$. Now (3.37) follows directly by taking the supremum in (3.40) over all $\alpha$ in $\mathscr{C}$. Finally let us notice that (3.38) follows easily by (3.41) being valid for all $\alpha \in \mathscr{C}$. This fact completes the proof.

The preceding strengthening of Theorem 3.1 may be applied in the setting of Theorem 3.3. In this way we obtain the following stronger version of Wiener's dominated ergodic theorem in exponential type Orlicz spaces.

Corollary 3.7. Let $(X, \mathscr{A}, \mu)$ be a Lebesgue space, and let $\mathscr{C}$ denote the topological group of all automorphisms in $(X, \mathscr{A}, \mu)$. Let us for given $\tau \in \mathscr{C}$ consider the sequence of linear operators $\left\{T_{n}^{\tau} \mid n \geq 1\right\}$ defined by:

$$
T_{n}^{\tau} f=\frac{1}{n} \sum_{j=0}^{n-1} f \circ \tau^{j}
$$

for all $f \in L^{1}(\mu)$ and all $n \geq 1$, and let us put $T_{\tau}^{*} f=\sup _{n \geq 1}\left|T_{n}^{\tau} f\right|$ for all $f \in$ $L^{1}(\mu)$. Then the following inequality is satisfied:

$$
\sup _{\tau \in \mathscr{C}}\left\|T_{\tau}^{*} f\right\|_{\psi_{p}} \leq C_{p} \cdot\|f\|_{\psi_{p}}
$$

for all $f \in L^{\phi_{p}}(\mu)$ with some $C_{p}>0$ and for given $1 \leq p<\infty$. Moreover we have:

$$
\sup _{\tau \in \mathscr{C}} \mu\left\{T_{\tau}^{*} f>\lambda \cdot\|f\|_{\psi_{p}}\right\} \leq C_{p} \cdot \exp \left(-D_{p} \cdot \lambda^{p}\right)
$$

for all $\lambda>0$ and all $f \in L^{\psi_{p}}(\mu)$ with some $C_{p}, D_{p}>0$ and for given $1 \leq p<\infty$.

Proof. Let $\sigma \in \mathscr{C}$ be an ergodic automorphism in $(X, \mathscr{A}, \mu)$, then by Theorem 3.3 we see that (3.36) in Theorem 3.6 is satisfied with $A=\left(a_{n k} \mid n, k\right.$ 
$\geq 1$ ) being a Toeplitz matrix given by $a_{n k}=1 / n$ for $k=1, \ldots, n$ with $n \geq 1$ and $a_{n k}=0$ otherwise. Thus (3.42) and (3.43) follow straightforward by (3.37) and (3.38) in Theorem 3.6. These facts complete the proof.

\section{REFERENCES}

[1] Atlagh, M. and Weber, M.. Théorèmes de densité pour des sommes de variables aléatoires, preprint (1993). To appear in the Proceedings of the Conference in Ergodic Theory and Probability, Columbus 1993.

[2] Conze, J. P., Convergence de moyennes ergodiques pour des sous-suites, Bull. Soc. Math. France, 35 (1973), 7-15.

[3] Dunford, N. and Schwartz, J. T., Linear Operators, Part 1: General Theory, Interscience Publ. Inc., New York, 1958.

[4] Furstenberg, H., Recurrence in Ergodic Theory and Combinatorial Number Theory, Princeton University Press, 1981.

[5] Garsia, A. M., Topics in Almost Everywhere Convergence, Lectures in Advanced Mathematics 4, Markham Publishing Company. 1970.

[6] Halmos, P. R., Lectures on Ergodic Theory, Math. Soc. Japan, 1956.

[7] Hoffmann-Jørgensen J., Function norms, Institute of Mathematics, University of Aarhus, Preprint Series, No. 40, (1990).

[8] Kolmogorov, A., Sur les fonctions harmoniques conjugées et les séries de Fourier, Fund. Math., 7 (1925), 23-28.

[9] Krengel, U., Ergodic Theorems, Walter de Gruyter \& Co., Berlin, 1985.

[10] Marcus, M. B. and Pisier, G., Stochastic processes with sample paths in exponential Orlicz spaces, Probability in Banach spaces V, Lecture Notes in Math., 1153 (1985), 328-358.

[11] Peškir, G., Best constants in Kahane-Khintchine inequalities in Orlicz spaces, Institute of Mathematics, University of Aarhus, Preprint Series, No. 10 (1992); J. Multivariate Anal., 45 (1993), 183-216.

[12] - Maximal inequalities of Kahane-Khintchine's type in Orlicz spaces, Institute of Mathematics, University of Aarhus, Preprints Series, No. 33 (1992); Math. Proc. Cambridge Philos. Soc., 115 (1994), 175-190.

[13] Petersen K., Ergodic Theory, Cambridge University Press. 1983.

[14] Sawyer, S., Maximal inequalities of weak type, Ann. Math., 84 (1966), 157-173.

[15] Stein, E. M., On limits of sequences of operators, Ann. Math., 74 (1961), 140-170.

[16] Weber, M., Une version fonctionelle du théoème ergodique ponctuel, C. R. Acad. Sci. Paris, t. 311, Série I, (1990), 131-133.

[17] - GC sets, Stein's elements and matrix summation methods, Prepublication. IRMA, No. 27, Strasbourg University, 1993.

[18] - Operateurs réguliers dans les espaces $L^{p}$, Séminaire de Probabilitiés XXVII, Lecture Notes in Math., 1557 (1993), 207-215.

[19] Zygmund, A., Trigonometric Series, Vol. 1, 11, Cambridge Univ. Press, New York, 2nd ed., 1959. 
Svend Erik Graversen

Institute of Mathematics, University of Aarhus

Ny Munkegade, 8000 Aarhus

Denmark

Goran Peškir

Institute of Mathematics, University of Aarhus

Ny Munkegade, 8000 Aarhus

Denmark

Department of Mathematics, University of Zagreb

Bijenička 30, 41000 Zagreb

Croatia

Michel Weber

I.R.M.A. Unité de Recherche associée C.N.R.S., 1

7, rue René Descartes, 67084 Strasbourg

France 